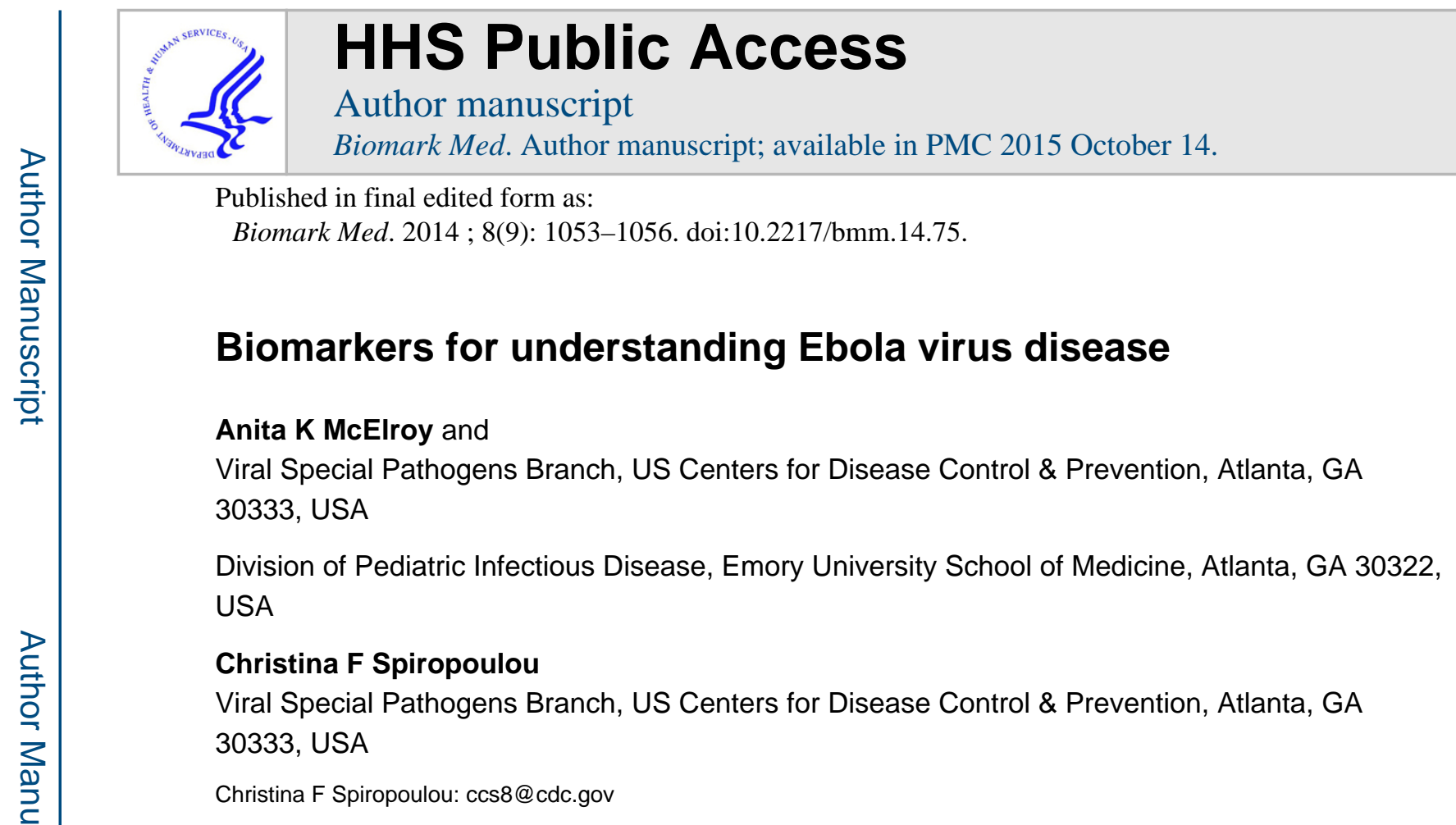

\title{
Keywords
}

ebola; biomarkers; cytokines; EVD

\section{The problem}

The Filoviridae family of negative-strand RNA viruses is feared for these viruses' ability to cause hemorrhage and death in infected individuals. Depending on the infecting species of virus, the case fatality rate can be anywhere from 30 to $90 \%$ [1]. Ebola virus (EBOV), Sudan virus (SUDV) and Bundibugyo virus (BDBV) are the three clinically significant species that cause Ebola virus disease (EVD). These viruses are typically found in sub-Saharan Africa, and cause sporadic outbreaks that can be brought under control with appropriate rapid public health responses. However, the recent emergence of EBOV in west Africa in early 2014 [2] has been challenging to contain, and has spread to three countries in the region so far. This outbreak, now the largest EBOV outbreak on record, is still ongoing at the time of writing this editorial.

Efforts by many investigators have led to the development of potential vaccines and therapeutics [3,4]. However, none of these have yet resulted in commercially available products, and are unlikely to do so in the near future due to the cost of developing novel products for human use and the challenges of conducting a clinical trial during an outbreak in a developing nation that lacks the necessary healthcare infrastructure. The most costeffective and timely option would be to identify an already approved drug that might be

Correspondence to: Christina F Spiropoulou, ccs8@edc.gov.

Financial \& competing interests disclosure

The authors have no relevant affiliations or financial involvement with any organization or entity with a financial interest in or financial conflict with the subject matter or materials discussed in the manuscript. This includes employment, consultancies, honoraria, stock ownership or options, expert testimony, grants or patents recived or pending or royalties.

No writing assistance was utilized in the production of this manuscript. 
effective at combating disease caused by EBOV and SUDV for off-label use by treating physicians. In order to identify currently licensed drugs that might be beneficial, we need to know more about the pathophysiology of the disease. Biomarker analyses could prove to be useful for this purpose.

\section{What we know}

\section{EBOV selectively targets antigen-presenting cells \& hepatocytes}

Two main cellular tropisms have been defined for EBOV and SUDV. The viruses readily infect cells of the monocyte/macrophage lineage, including dendritic cells and hepatocytes. Other infected cell types, including endothelial cells, have been noted in animal models and autopsy specimens but are typically found late in infection when viral loads are high. Consistent with the theory that infection of antigen-presenting cells and hepatocytes is responsible for the majority of EBOV's pathogenesis is the finding that mice, which are naturally resistant to EBOV, can develop severe, lethal and human-like diseases if they are engrafted with human CD34+ hematopoietic stem cells and autologous human liver and thymus [Bird B, Unpublished data].

\section{Biomarkers can indicate the severity of the disease as well as its putative mechanisms}

One way in which investigators have attempted to study EVD in humans is by measuring various analytes in serum or plasma specimens obtained from infected individuals. This type of analysis has been done in humans for EBOV, SUDV and BDBV.

\section{Ebola virus}

While EBOV research is ever expanding, the currently available biomarker data are limited. Two separate investigations used ELISAs or multiplex assays of serum and plasma samples, as well as RT-PCR from PBMC RNA, to analyze biomarkers in EBOV-infected individuals from six different outbreaks. They reported increased levels of IL-1 $\beta$, IL1-RA, IL-2, IL-6, IL-8, IL-10, MIP-1a, MIP-1 $\beta$, MCP-1, MCSF, MIF, IP-10, GRO- $a$, eotaxin, neopterin, sTNF-RI, TNF- $\alpha$, sFasL, sFas, IFN- $\alpha$ and IFN- $\gamma$ [5-8]. These data gave rise to the concept that excessive immune activation, or 'cytokine storm', upon EBOV infection is detrimental to infected individuals. Unfortunately, these findings are relatively nonspecific, because there is no way to determine the contribution of each cytokine to the pathophysiology of EVD, and, as discussed below, contradictory data exist on the role of cytokines in EVD.

In addition to the increased sFasL and sFas levels seen in the above-mentioned studies, direct analyses of infected PMBCs found DNA laddering and lymphopenia as well as increased CD95 (Fas) staining by flow cytometric evaluation. Together, these findings demonstrate that lymphocyte apoptosis is induced by EBOV infection. Moreover, lymphocyte apoptosis was associated with fatal outcomes $[7,8]$.

Studies of individuals with asymptomatic EBOV infections complicate the 'cytokine storm' concept, however. These studies demonstrate pro-inflammatory cytokine level magnitudes higher than those seen in symptomatic individuals, but only in asymptomatic samples collected the week following virus exposure [9]. Serial sampling of these asymptomatic 
individuals shows rapid declines in pro-inflammatory cytokine levels even before EBOV is fully cleared or an antibody response develops. Therefore, these cytokine responses in themselves are not detrimental to the host. Rather, the timing of the response and/ or its subsequent downregulation seems to be important for survival. In a further study of PMBC RNA from the same asymptomatic patients, the investigators identified biomarkers of T-cell activation: $I L-2, I L-4, C D-28, C D-40 L$ and $C T L A-4$ were all upregulated immediately after the initial pro-inflammatory cytokine burst [10]. Additionally, using RNA biomarkers of apoptosis and T-cell activity, including perforin, Fas, FasL and $I F N-\gamma$, they found evidence of contraction of the T-cell population occurring at the same time as antibody development in these patients, consistent with an appropriate adaptive response.

\section{Bundibugyo virus}

Even fewer biomarker studies have been conducted with BDBV. One small study found that samples from fatal cases had increased levels of IFN- $\alpha 2$, IFN- $\gamma$ and IL-10 [11]; these findings were consistent with what has been reported for EBOV.

\section{Sudan virus}

Prior to the current EBOV outbreak in West Africa, the Gulu outbreak of SUDV in 20002001 was the largest outbreak of EVD ever recorded [12]. An international team responded, and serial specimens were saved from many of the affected patients, making hundreds of samples available for testing. Much of what we know about EVD has come from analyses of these specimens. Levels of aspartate aminotransferase and ${ }_{\mathrm{D}}$-dimer were elevated in patients who had a fatal outcome [13]. These biomarker elevations are consistent with liver tropism, and indicate possible contribution of coagulopathy to EVD. Nitric oxide, a reactive oxygen species that could be associated with the hypotension/shock that is seen in EVD, was also elevated in fatal cases [14].

The large number of available specimens from the Gulu outbreak permitted the analysis of cytokines and chemokines over time in serial specimens obtained from 150 patients. The analyses showed that IL-6, IL-8, IL-10 and MIP-1 $\beta$ were higher in patients with fatal outcomes, but IFN-a was higher in patients who survived [15]. The idea that the "cytokine storm' is detrimental, therefore, is quite an over simplification, since IFN-a, a notable proinflammatory antiviral cytokine, was associated with survival, while IL-10, a cytokine with known anti-inflammatory properties, was associated with a fatal outcome. Rather, an overall immune dysregulation appears to exist at the cytokine level, followed by reduced numbers of total and activated CD8 T cells in SUDV-infected patients who have a fatal outcome [14].

The massive growth in the number of commercially available multiplex assays for biomarker analysis permitted us to go back to these same serum specimens and conduct novel biomarker assays, since these can be performed with small amounts of patient specimens. In our recent study, we reported that SCD40L was associated with survival [16], a finding that suggests the possible role of activated platelets in promoting survival, and/or the possibility that SCD40L can stimulate antigen-presenting cells to overcome SUDVinduced inhibition of these cells [17]. This finding in particular has significant implications for EVD management in patients, and suggests two potential therapies: platelet 
administration (already indicated, since many patients are thrombocytopenic), or recombinant CD-40L administration. The latter strategy has already been used in patients with hyper-IgM, a disorder in which CD-40L is missing or defective [18].

Additionally, we found that thrombomodulin levels were elevated in patients with a fatal outcome, as well as in patients who had hemorrhagic manifestations [16]. Thrombomodulin functions in endothelial stability when it is present on the endothelium rather than freefloating in the serum, and also in inhibiting the coagulation pathway. Our finding underscores the complex interaction between the endothelium, the immune system and the coagulation pathway. In fact, therapies that provide increased stability to the endothelium will likely benefit patients not only by improving hemodynamic stability, but also by decreasing the likelihood of hemorrhagic manifestations.

\section{What we need to know \& how to get there}

Absolutely critical to improving our ability to combat EVD is a thorough understanding of the mechanisms of this disease. A significant amount of aberrant immune signaling is detected in fatal cases, and this is accompanied by the lack of an effective adaptive response. Therefore, analyses of the cellular immune response in samples from both symptomatic and asymptomatic cases will be essential. Clearly, some aspects of the immune response are beneficial to the host, so defining exactly which aspects these are, and the kinetics of when they need to occur, will be very helpful for our understanding of EVD pathogenesis. Acquiring this knowledge will require serial sampling of patients during outbreaks, proper handling and storage of specimens and qualified personnel and facilities to carry out the studies. Coordination between clinicians and researchers will be necessary to ensure that this is done in an ethically sound manner. Biomarker analyses of patient specimens has given us an immense amount of information about EVD, but in order to go full circle, we need to use this information to guide future studies with the ultimate goal of improving patient care, mitigating disease and saving lives.

\section{References}

1. Sanchez, A.; Geisbert, T.; Feldmann, H. Filoviridae: Marburg and Ebola Viruses. 5th Edition. Philadelphia, PA, USA: Wolters kluwer/Lippincott Williams \& Wilkins; 2007.

2. Baize S, Pannetier D, Oestereich L, et al. Emergence of Zaire Ebola virus disease in Guinea preliminary report. N. Engl. J. Med. 2014 (Epub ahead of print).

3. Marzi A, Feldmann H. Ebola virus vaccines: an overview of current approaches. Expert Rev. Vaccines. 2014; 13(4):521-531. [PubMed: 24575870]

4. Wong G, Qiu X, Olinger GG, Kobinger GP. Postexposure therapy of filovirus infections. Trends Microbiol. 2014 (Epub ahead of print).

5. Baize S, Leroy EM, Georges AJ, et al. Inflammatory responses in Ebola virus-infected patients. Clin. Exp. Immunol. 2002; 128(1):163-168. [PubMed: 11982604]

6. Villinger F, Rollin PE, Brar SS, et al. Markedly elevated levels of interferon (IFN)-gamma, IFNalpha, interleukin (IL)-2, IL-10, and tumor necrosis factor-alpha associated with fatal Ebola virus infection. J. Infect. Dis. 1999; 179(Suppl. 1):S188-S191. [PubMed: 9988183]

7. Wauquier N, Becquart P, Padilla C, Baize S, Leroy EM. Human fatal Zaire Ebola virus infection is associated with an aberrant innate immunity and with massive lymphocyte apoptosis. PLoS Negl. Trop. Dis. 2010; 4(10):e837. [PubMed: 20957152] 
8. Baize S, Leroy EM, Georges-Courbot MC, Capron M, et al. Defective humoral responses and extensive intravascular apoptosis are associated with fatal outcome in Ebola virus-infected patients. Nat. Med. 1999; 5(4):423-426. [PubMed: 10202932]

9. Leroy EM, Baize S, Volchkov VE, et al. Human asymptomatic Ebola infection and strong inflammatory response. Lancet. 2000; 355(9222):2210-2215. [PubMed: 10881895]

10. Leroy EM, Baize S, Debre P, Lansoud-Soukate J, Mavoungou E. Early immune responses accompanying human asymptomatic Ebola infections. Clin. Exper. Immunol. 2001; 124(3):453460. [PubMed: 11472407]

11. Gupta M, MacNeil A, Reed ZD, Rollin PE, Spiropoulou CF. Serology and cytokine profiles in patients infected with the newly discovered Bundibugyo ebolavirus. Virology. 2012; 423(2):119124. [PubMed: 22197674]

12. Okware SI, Omaswa FG, Zaramba S, et al. An outbreak of Ebola in Uganda. Trop. Med. Int. Health. 2002; 7(12):1068-1075. [PubMed: 12460399]

13. Rollin PE, Bausch DG, Sanchez A. Blood chemistry measurements and d-dimer levels associated with fatal and nonfatal outcomes in humans infected with Sudan Ebola virus. J. Infect. Dis. 2007; 196(Suppl. 2):S364-S371. [PubMed: 17940972]

14. Sanchez A, Lukwiya M, Bausch D, et al. Analysis of human peripheral blood samples from fatal and nonfatal cases of Ebola (Sudan) hemorrhagic fever: cellular responses, virus load, and nitric oxide levels. J. Virol. 2004; 78(19):10370-10377. [PubMed: 15367603]

15. Hutchinson KL, Rollin PE. Cytokine and chemokine expression in humans infected with Sudan Ebola virus. J. Infect. Dis. 2007; 196(Suppl. 2):S357-S363. [PubMed: 17940971]

16. McElroy AK, Erickson BR, Flietstra TD, et al. Ebola hemorrhagic fever: novel biomarker correlates of clinical outcome. J. Infect. Dis. 2014 (Epub ahead of print).

17. Mahanty S, Hutchinson K, Agarwal S, McRae M, Rollin PE, Pulendran B. Cutting edge: impairment of dendritic cells and adaptive immunity by Ebola and Lassa viruses. J. Immunol. 2003; 170(6):2797-2801. [PubMed: 12626527]

18. Jain A, Kovacs JA, Nelson DL, et al. Partial immune reconstitution of X-linked hyper IgM syndrome with recombinant CD40 ligand. Blood. 2011; 118(14):3811-3817. [PubMed: 21841160] 